\title{
Breast Cancer Detection by Preoperative Imaging in Reduction Mammaplasty Patients : A Single Center Study of 918 Patients
}

\section{Merkkola-von Schantz, Paivi A.}

2017-08

Merkkola-von Schantz , P A , Kauhanen, S M C, Jahkola , T A, Krogerus , L \& Hukkinen, K S 2017 , ' Breast Cancer Detection by Preoperative Imaging in Reduction Mammaplasty Patients : A Single Center Study of 918 Patients ' , World Journal of Surgery, vol. 41 , no. 8 , pp. 2013-2019 . https://doi.org/10.1007/s00268-017-3920-z

http://hdl.handle.net/10138/237620

https://doi.org/10.1007/s00268-017-3920-z

publishedVersion

Downloaded from Helda, University of Helsinki institutional repository.

This is an electronic reprint of the original article.

This reprint may differ from the original in pagination and typographic detail.

Please cite the original version. 


\title{
Breast Cancer Detection by Preoperative Imaging in Reduction Mammaplasty Patients: A Single Center Study of 918 Patients
}

\author{
Päivi A. Merkkola-von Schantz ${ }^{1} \cdot$ Susanna M. C. Kauhanen $^{1}$ Tiina A. Jahkola ${ }^{1}$. \\ Leena A. Krogerus ${ }^{2} \cdot$ Katja S. Hukkinen ${ }^{3}$
}

Published online: 2 March 2017

(C) Société Internationale de Chirurgie 2017

\begin{abstract}
Background The role of preoperative imaging and the usability of different imaging modalities is highly variable and controversial in reduction mammaplasty patients. Our study describes the imaging process in a single center in regard to modality selection, age and timing, and of the association between imaging and histopathological findings in reduction mammaplasty specimens.

Methods Nine hundred eighteen women, who underwent reduction mammaplasty during 1.1.2007-31.12.2011, were retrospectively reviewed for demographics, preoperative imaging, further preoperative examinations, and pathology reports.

Results Preoperative imaging had been conducted for $89.2 \%(n=819)$ of the patients. In $49(6.0 \%)$ patients, suspicious preoperative imaging led to further examinations revealing 2 high-risk lesions (atypical ductal hyperplasia $(\mathrm{ADH})$, lobular carcinoma in situ (LCIS)), and 2 cancers preoperatively. Postoperatively abnormal histopathology specimens were revealed in $88(10.4 \%)$ patients. The incidence of high-risk lesions was $5.5 \%(n=47)$, and the incidence of cancer was 1.2\% $(n=10)$. Preoperative imaging was normal (BI-RADS 1 and BI-RADS 2$)$ in $80.8 \%$ of these patients. The sensitivity of the preoperative imaging for cancer detection was $20.0 \%$, and the specificity was $100.0 \%$.

Conclusions Preoperative imaging and further examinations do not sufficiently detect malignant or cancer riskincreasing findings. Therefore, histopathological analysis of reduction mammaplasty specimens seems mandatory.
\end{abstract}

Päivi A. Merkkola-von Schantz

paivi.merkkola-vonschantz@hus.fi

1 Department of Plastic and Reconstructive Surgery, University of Helsinki and Helsinki University Hospital, P.O. Box 266, 00029 Helsinki, Finland

2 Department of Pathology, University of Helsinki and Helsinki University Hospital, P.O. Box 800, 00029 Helsinki, Finland

3 Department of Radiology, University of Helsinki and Helsinki University Hospital, P.O. Box 263, 00029 Helsinki, Finland

\section{Introduction}

Reduction mammaplasty is a common procedure in plastic surgery. Indications for this surgery are symptomatic macromastia, breast asymmetry, and contralateral breast symmetrisation during or after breast cancer surgery. Breast cancer is the most frequent cancer among women, the lifetime risk being 1 in 8 . It is thus unsurprising that incidental cancers, in situ findings, and benign breast disease demonstrating increased risk of breast cancer are revealed in the process of reduction mammaplasty.

The incidence of occult breast cancer in reduction mammaplasty specimens has been under study in several countries with incidences ranging from 0.05 to 4.5 percent 
Table 1 BI-RADS classification

\begin{tabular}{lll}
\hline Category & Definition & Likelihood of cancer \\
\hline BI-RADS 0 & Incomplete & N/A \\
BI-RADS 1 & Negative & Essentially $0 \%$ \\
BI-RADS 2 & Benign & Essentially $0 \%$ \\
BI-RADS 3 & Probably benign & $>0 \%$, but $\leq 2 \%$ \\
BI-RADS 4 & Suspicious & $>2 \%$, but $<95 \%$ \\
BI-RADS 5 & Highly suggestive of malignancy & $\geq 95 \%$ \\
BI-RADS 6 & Known biopsy-proven malignancy & N/A \\
\hline
\end{tabular}

[1-9]. Studies also indicate that women with benign breast disease, commonly detected in reduction mammaplasty specimens, are at higher risk for breast cancer [2, 3, 10-17].

The question of routine preoperative imaging in reduction mammaplasty is an ongoing one, which we have not yet come to a consensus. Mammogram is variously recommended for different age groups [1, 3, 5, 7, 8, 12, 18-21].

The present study aims to retrospectively describe the use of different imaging modalities. The association between preoperative imaging, needle biopsies, and final histopathological findings in reduction mammaplasty patients is described.

\section{Materials and methods}

A total of 1255 women underwent reduction mammaplasties during 1.1.2007-31.12.2011. Patients with previous history of breast cancer were excluded and the remaining patients amounting to 918 . The indications for the surgery were symptomatic macromastia or asymmetry of the breasts. Unilateral procedures were performed in 35 cases due to congenital or postoperative asymmetry. Findings were recorded per individual and not per breast. Retrospective electronic and paper records were retrieved. Demographic data, results of preoperative imaging, operative and pathology reports, and postoperative follow-up were recorded. The study was approved by the University Hospital Research Board.

During the study period, imaging protocols varied. Ultrasound, mammogram, or both imaging modalities were conducted depending which was the imaging site, breast density, and age. Some patients were referred to conduct imaging in the private sector or in primary healthcare centers. Some patients did not undergo any preoperative imaging. The different approaches to imaging were due to present routines, and thus, the groups were not designed for research purposes.

Preoperative imaging findings were retrospectively classified according to the American College of Radiology Breast Imaging Reporting and Data System (BI-RADS)
[22]. This classification is presented in Table 1. In our study, BI-RADS 1 and BI-RADS 2 were categorized as normal breast imaging findings and BI-RADS 3 and BI-RADS 4 as suspicious of malignancy. Breast density was retrospectively analyzed for patients with malignant postoperative histopathology according to BI-RADS lexicon [22].

For histopathological analysis, reduction mammaplasty specimens were weighed, formalin fixed, and cut into $1-\mathrm{cm}$ slices that were palpated for masses and for areas of increased density. Samples for blocks were taken from macroscopically suspicious areas and analyzed histopathologically. The number of blocks per breast varied between 4 and 20, 5 being the most usual amount.

We categorized abnormal histopathological findings in reduction mammaplasty specimens based on a consensus statement outlined by the Cancer Committee of the College of American Pathologists in 1985 and incorporated the 1998 consensus statement update [16]. In short, high-risk lesions included atypical ductal hyperplasia (ADH), atypical lobular hyperplasia (ALH), and lobular carcinoma in situ (LCIS). Invasive cancer and ductal carcinoma in situ (DCIS) were classified as cancer findings.

We retrospectively studied if the findings in preoperative imaging associated with histopathological diagnosis of the specimens. Those patients who had malignant postoperative histopathology had their mammogram reviewed and re-analyzed by radiologist (K.H.) with 10 years of breast imaging experience. We also registered the time frame in which patients had completed preoperative imaging prior to surgery, and 6 months or less was considered as a cutoff according to the present recommendation.

Descriptive statistics were reported as the mean value and range between minimum and maximum. Pearson's Chi-squared test was applied in bivariate analyses with categorical variables. Two-sample $t$ test and analysis of variance were used when patient age was compared between patient groups. The sensitivity of preoperative imaging and diagnosis was calculated as cancers detected preoperatively compared to all cancers diagnosed in reduction mammaplasty specimens. The specificity was calculated as patients with normal preoperative imaging compared to patients without cancer in their specimens. 
Table 2 Preoperative imaging

\begin{tabular}{lcl}
\hline Imaging modality & Patients $(\%)$ & Mean age and range \\
\hline Mammogram alone & $250(27.2 \%)$ & $47.0(18-73)$ \\
Ultrasound alone & $15(1.6 \%)$ & $21.5(18-26)$ \\
Both modalities & $554(60.3 \%)$ & $43.8(18-79)$ \\
No imaging & $99(10.8 \%)$ & $43.2(16-68)$ \\
\hline
\end{tabular}

\section{Results}

A total of 918 women underwent reduction mammaplasty with a mean age of 44.3 years (range 16-79 years) and a mean body mass index of 27.7 (range 19.0-50.5). Preoperative imaging had been conducted for $89.2 \%$ of the patients $(n=819)$. The different imaging modalities and the number of patients are presented in Table 2. The mean age of the patients with normal (BI-RADS 1 and BI-RADS 2) imaging findings did not statistically differ from patients with imaging suspicious of malignancy (BI-RADS 3 and BI-RADS 4).

\section{Association between BI-RADS class of mammogram and ultrasound}

Among the patients with both imaging modalities ( $n=554$ ), BI-RADS classes of mammogram and ultrasound coincided in $536(96.8 \%)$ of the patients. For 18 patients (aged 32-67 years), additional ultrasound revealed suspicious lesions, which were undetectable in mammogram. These lesions were biopsied $(n=15)$ with benign results, or surgical open biopsy $(n=2)$ was performed simultaneously with reduction mammaplasty. Final histopathology revealed high-risk lesions in 5 patients. One patient had no further work-up despite BI-RADS 4 class in the ultrasound (left breast), and the final histopathology revealed DCIS in both breasts, sized $7 \mathrm{~mm}$ (right breast) and $2.5 \mathrm{~mm}$ (left breast).

\section{Preoperative further examinations}

In total, BI-RADS 3 and BI-RADS 4 category in preoperative imaging was found in $56(6.8 \%)$ patients of the 819 imaged. In 12 of these patients, no further examinations were performed. In 49 of $819(6.0 \%)$ patients, imaging led to further examinations. The mean age of these patients was 45.3 years (range 23-67 years). Mammographic magnification of suspicious area had been conducted for 9 patients. Figure 1 shows the process of patients needing biopsies. In total, further examinations revealed 2 cancers (48 and 58 years), 1 ADH (50 years), and 1 LCIS
(56 years) finding. Table 3 demonstrates features and treatment of 2 preoperatively diagnosed cancer findings.

\section{Histopathology}

Histopathologically abnormal findings in reduction mammaplasty specimens were revealed in $88(10.4 \%)$ patients. The incidence of high-risk lesions was $5.5 \%(n=47)$. In $69(7.5 \%)$ patients, no sample was taken for histopathological analysis. In patients with abnormal histopathology, preoperative imaging had been conducted for $78(88.6 \%)$ patients and no imaging was performed for $10(11.4 \%)$ patients. Preoperative imaging was normal (BI-RADS 1 and BI-RADS 2) in $80.8 \%$ and suspicious of malignancy (BI-RADS 3 and BI-RADS 4) in 19.2\% of these patients.

Among patients $(n=56)$ with imaging suspicious of malignancy (BI-RADS 3 and BI-RADS 4), reduction mammaplasty specimens revealed abnormal histopathological findings in $27.3 \%$ and normal findings in $72.7 \%$ of the patients. One patient had no histopathological analysis of reduction mammaplasty specimen despite suspicious imaging. During the study period, no mention of subsequent oncological incident was found with this patient.

Final histopathology revealed $10(1.2 \%)$ patients with invasive cancer or DCIS. The mean age of the patients was 55.5 years (range 48-67 years). The features of preoperatively undetected cancer findings are demonstrated in Table 4.

Preoperative imaging of the 10 patients with malignant histopathology was retrospectively searched for, and 8 were retrieved and re-analyzed by an experienced radiologist. None of the previously undetected cancer findings could be identified retrospectively.

\section{Sensitivity and specificity of imaging}

The sensitivity of the preoperative imaging was $20.0 \%$, considering that the final histopathology encompasses only the operated part of the breast. There were no false-positive preoperative biopsy findings leading to specificity of $100.0 \%$.

\section{Timing of imaging}

The date of imaging could be retrieved from patient records in 738 cases. The number of patients with conducted imaging within 6 months prior to surgery was 699 (94.7\%), and the number of patients with older imaging was 39 (5.3\%). Abnormal histopathological findings were detected in $9.7 \%$ in the timely imaged group and in $12.8 \%$ in the group with older imaging (ns). 


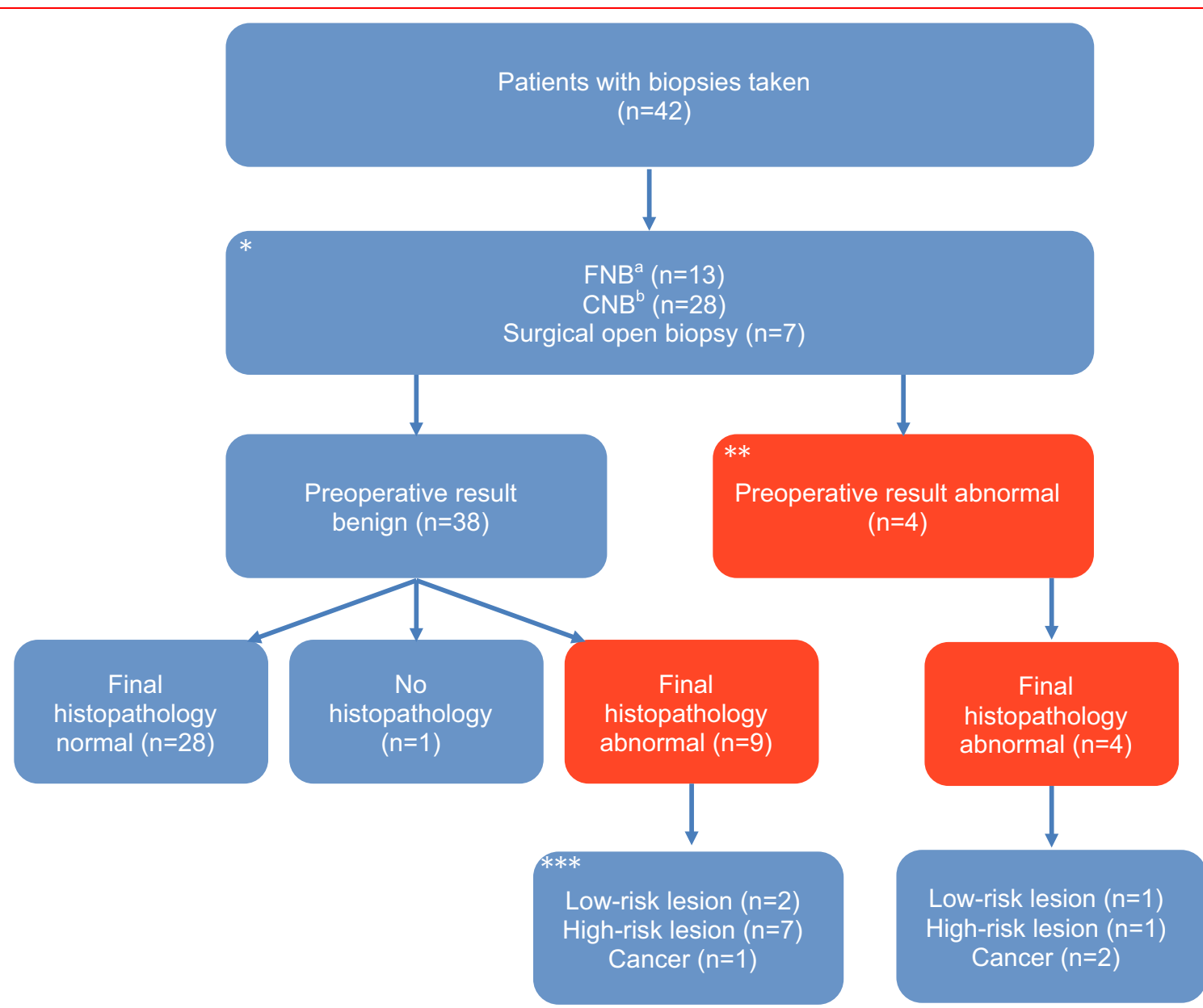

Fig. 1 Illustration of the patients needing biopsies. Note: ${ }^{\mathrm{a}} \mathrm{FNB}$ Fine needle biopsy, ${ }^{\mathrm{b}} \mathrm{CNB}$ Core needle biopsy. ${ }^{*}$ Six patients needed 2 simultaneous examinations, ${ }^{* *}$ Cancer $(n=2)$, ADH $(n=1)$, LCIS $(n=1),{ }^{* * *}$ One patient had 2 simultaneous lesions

Table 3 Preoperatively diagnosed cancer findings

\begin{tabular}{|c|c|c|c|c|c|}
\hline Patient & Imaging & Tumor size in imaging & $\begin{array}{l}\text { Needle } \\
\text { biopsy }\end{array}$ & Treatment & $\begin{array}{l}\text { Tumor size in } \\
\text { histopathology }\end{array}$ \\
\hline $\begin{array}{l}\text { Patient } \\
1\end{array}$ & $\begin{array}{l}\mathrm{MMG}^{\mathrm{a}}(\mathrm{BI}-\mathrm{RADS} 4), \mathrm{US}^{\mathrm{b}}(\mathrm{BI}- \\
\quad \text { RADS 4) D2 }\end{array}$ & 14 mm (MMG, US) & $\mathrm{CNB}^{\mathrm{d}}$ & $\begin{array}{l}\text { Oncoplastic resection, } \mathrm{SNB}^{\mathrm{e}}, \\
\text { contralateral reduction } \\
\text { mammaplasty }\end{array}$ & $13 \mathrm{~mm}$ \\
\hline $\begin{array}{l}\text { Patient } \\
\quad 2\end{array}$ & $\begin{array}{l}\text { MMG (BI-RADS 2, 4), US (BI- } \\
\text { RADS 4) D1 }\end{array}$ & $\begin{array}{l}11 \mathrm{~mm}(\mathrm{MMG}) \\
10 \times 7 \times 7 \mathrm{~mm}(\mathrm{US})\end{array}$ & $\mathrm{CNB}$ & $\begin{array}{l}\text { Oncoplastic resection, SNB, } \\
\text { contralateral reduction } \\
\text { mammaplasty }\end{array}$ & $12 \mathrm{~mm}$ \\
\hline
\end{tabular}

\footnotetext{
${ }^{\text {a }} M M G$ mammogram

${ }^{\mathrm{b}}$ US ultrasound

${ }^{\mathrm{c}} D$ breast density

d $C N B$ core needle biopsy

e $S N B$ sentinel node biopsy
}

\section{Discussion}

Preoperative imaging before reduction mammaplasty remains controversial, as no consensus exists. Our study allows analysis of the imaging process in regard to modality selection, age and timing, and of the association between imaging and histopathological findings in reduction mammaplasty specimens.

In our study, the imaging protocol differed between imaging sites. One imaging center conducted mammogram 
Table 4 Preoperatively undetected cancer findings in reduction mammaplasty specimens

\begin{tabular}{|c|c|c|c|c|c|c|}
\hline Patient & Age & $\begin{array}{l}\text { Imaging } \\
\text { modality }\end{array}$ & $\begin{array}{l}\text { Further } \\
\text { examinations }\end{array}$ & Result & $\begin{array}{l}\text { Histopathological diagnosis of the } \\
\text { specimen }\end{array}$ & Size of cancer \\
\hline 1 & 51 & $\mathrm{MMG}^{\mathrm{a}}, \mathrm{US}^{\mathrm{b}} \mathrm{D} 2^{\mathrm{c}}$ & $\mathrm{FNB}^{\mathrm{d}}, \mathrm{CNB}^{\mathrm{e}}$ & Benign & Carcinoma ductale & $40 \mathrm{~mm}$ \\
\hline 2 & 51 & MMG, US D3 & None & - & DCIS $^{\mathrm{f}}$ (both breasts) & $7 \mathrm{~mm}$ (right) $2,5 \mathrm{~mm}$ (left) \\
\hline 3 & 49 & MMG, US D3 & None & - & DCIS (right) Carcinoma lobulare (left) & $2 \mathrm{~mm}$ (right) 7 lesions, $2-6 \mathrm{~mm}$ (left) \\
\hline 4 & 50 & MMG, US D2 & None & - & Carcinoma lobulare, DCIS & 7 mm (cancer) unknown (DCIS) \\
\hline 5 & 67 & MMG D1 & None & - & Carcinoma ductale & $7 \mathrm{~mm}$ \\
\hline 6 & 57 & MMG D3 & None & - & DCIS & $2 \mathrm{~mm}$ \\
\hline 7 & 62 & MMG D2 & None & - & DCIS & $11 \mathrm{~mm}+7 \mathrm{~mm}$ \\
\hline 8 & 62 & None & None & - & DCIS & $2 \mathrm{~mm}$ \\
\hline
\end{tabular}

a $M M G$ mammogram

${ }^{\mathrm{b}}$ US ultrasound

c $D$ breast density

${ }^{d}$ FNB fine needle biopsy

e $C N B$ core needle biopsy

${ }^{\mathrm{f}}$ DCIS ductal carcinoma in situ

and ultrasound for the majority of the patients regardless of age. Another center conducted ultrasound for women under 28 years and mammogram for older with additional ultrasound in case of dense breasts or suspicious findings in mammogram. A group of patients were asked to conduct imaging in the private sector or in primary healthcare centers. In $10.8 \%$ of the patients, with a mean age of 43.2 years, neither of the imaging modalities was completed or the information about imaging could not be confirmed. Younger patients may symbolize low-cancerrisk patients, but this does not explain the lack of imaging in older patients. Pending information on imaging may be due to a large amount of patients who conduct imaging in the private sector with no mention about imaging in the patient records. This focuses attention on the importance of a preoperative routine.

In the literature, variation exists between imaging protocols in different countries. In the UK, $92 \%$ of breast surgeons and $41 \%$ of plastic surgeons routinely performed radiological screening for reduction mammaplasty patients. The majority chose age as an indicator for screening [21]. In the Netherlands [18], only 3\% of the responders to a survey routinely required preoperative mammogram, and only 1 responder routinely required preoperative ultrasound. In general, preoperative mammogram is variously recommended from the age of 30 [12], from the age of $40[3,5,18,23,24]$, or for patients over the age of 50 [19]. In our study, patients were imaged in all age groups, the mean age of these patients being over 40 years.

Reduction mammaplasty changes the architecture of the breast. In case of incidental cancer, breast-conserving options may be limited. Therefore, emphasis should be placed on preoperative diagnosis [23, 24]. In our study, $80.8 \%$ of the patients with abnormal findings in reduction mammaplasty specimens had normal preoperative imaging. Similarly, others $[3,5-7,20]$ have noticed that incidental discovery of atypical hyperplasia, LCIS, or cancer were not associated with abnormal imaging. Moreover, only 2 out of 10 cancers in our study were detected preoperatively. It seems that preoperative imaging does not sufficiently detect high-risk or cancer findings. Therefore, histopathological analysis of reduction mammaplasty specimens seems difficult to bypass.

Among the preoperatively undetected cancers, 1 patient (carcinoma ductale $40 \mathrm{~mm}$ ) had fine and core needle biopsies taken with benign results. Either biopsies were targeted incorrectly, or more likely preoperative histopathology was suboptimized. In 3 patients, both mammogram and ultrasound were unable to detect cancer or DCIS preoperatively despite bilateral malignancies in 2 of them. Growth pattern of DCIS and lobular cancer, as well as small size, may explain why these lesions were undetectable. In 3 patients with malignant outcome, preoperative mammogram alone, with breast densities varying from D1 to D3, was conducted. In theory, the false-negative ductal cancer, sized $7 \mathrm{~mm}$, might have been found with additional ultrasound. In 1 patient, no preoperative imaging was conducted, which precludes the possibility of preoperative diagnostics. Nevertheless, small invasive cancers, DCIS, or high-risk lesions may remain undetected with all imaging modalities, including MRI. In our study, the sensitivity of the imaging was $20.0 \%$. It can be explained by small size of undetected cancers. The 
specificity in our study was $100.0 \%$. There were no falsepositive cancers.

Our study revealed 18 patients with incoherent imaging. Despite normal mammogram, ultrasound was performed and showed BI-RADS 4 unexpectedly. Eventually, 33.3\% of the patients had either DCIS or a high-risk lesion in the specimens. Although actual cancer findings in the reduction mammaplasty specimens were rare in this patient group, a considerable amount of findings indicating increased risk of breast cancer were detected. Benign breast disease is an important predictor of future breast cancer risk [10-17]. High-risk lesions, including ADH and ALH, cause moderately increased risk (4.0-5.0 times), and LCIS causes markedly increased risk (8.0-10.0 times) of breast cancer [16]. In screening situations [25], patients with a history of ADH or ALH, or LCIS may benefit from adjunct (ultrasound or MRI) screening due to lower mammogram specificity and higher interval cancer rates. However, in our study, a substantial amount of work with false-positive imaging raises the question of the use of routine ultrasound in combination with mammogram, as opposed to ultrasound only in dense breasts or additionally to suspicious mammogram. Based on our results, it remains to be elucidated if both ultrasound and mammogram are needed.

The importance of screening mammogram has been debated lately and guidelines re-assessed. Also, there are differences in target age between countries. Currently, routine mammographic screening is not recommended for women under the age of 40 . Sensitivity of mammogram is lower among young women and in dense breasts [26]. However, breast cancers in very young women are typically aggressive [27], and DCIS in young women is often multifocal and multicentric [28]. These studies support preoperative imaging also in very young women. Based on this, prior to major breast surgery changing the architecture of the breast, we recommend preoperative ultrasound for women under 30 years of age in our unit, and mammogram is recommended for patients older than that. There are no national guidelines available. In our study, a large number of patients $(n=819)$ conducted imaging with only 2 cancers and 2 high-risk lesions detected preoperatively. Still, these operations could be planned and performed as oncoplastic resections, which supports the role of preoperative imaging. On the other hand, all other abnormalities went undetected in the imaging. This highlights the value of histopathological analysis as the method to detect cancer and risk-increasing lesions.

We acknowledge some limitations to our study. In a large institution and between facilities, breast imaging and the threshold to conduct additional imaging or examinations may vary. Also, patients requiring mastectomy or more extensive oncological treatment may have been referred to Breast Unit, and therefore, the data are missing from our material. The study is retrospective, which compels us to rely on record keeping of others. The time period between surgery and data collection is limited. It remains uncertain, if the unoperated part of the breast contains cancer or risk-increasing findings. Therefore, the sensitivity of mammogram is calculated for the operated part of the breast, and the true sensitivity may be even lower. In 99 patients, it was not possible to discern between missing preoperative imaging and missing data.

In conclusion, emphasis should be placed on the quality and documentation of preoperative evaluation of reduction mammaplasty patients. To date, preoperative imaging and further examinations do not sufficiently detect cancer or high-risk lesions; therefore, histopathological evaluation of reduction mammaplasty specimens seems mandatory.

\section{Compliance with ethical standards}

Conflicts of interest All authors have no conflicts of interest to declare.

\section{References}

1. Hassan FE, Pacifico MD (2012) Should we be analysing breast reduction specimens? A systematic analysis of over 1,000 consecutive cases. Aesthetic Plast Surg 36:1105-1113

2. Clark CJ, Whang S, Paige KT (2009) Incidence of precancerous lesions in breast reduction tissue: a pathologic review of 562 consecutive patients. Plast Reconstr Surg 124:1033-1039

3. Ambaye AB, MacLennan SE, Goodwin AJ et al (2009) Carcinoma and atypical hyperplasia in reduction mammaplasty: increased sampling leads to increased detection. A prospective study. Plast Reconstr Surg 124:1386-1392

4. Boice JD Jr, Persson I, Brinton LA et al (2000) Breast cancer following breast reduction surgery in Sweden. Plast Reconstr Surg 106:755-762

5. Colwell AS, Kukreja J, Breuing KH et al (2004) Occult breast carcinoma in reduction mammaplasty specimens: 14-year experience. Plast Reconstr Surg 113:1984-1988

6. Kakagia D, Fragia K, Grekou A et al (2005) Reduction mammaplasty specimens and occult breast carcinomas. Eur J Surg Oncol 31:19-21

7. Slezak S, Bluebond-Langner R (2011) Occult carcinoma in 866 reduction mammaplasties: preserving the choice of lumpectomy. Plast Reconstr Surg 127:525-530

8. Freedman BC, Smith SM, Estabrook A et al (2012) Incidence of occult carcinoma and high-risk lesions in mammaplasty specimens. Int J Breast Cancer 2012:145630

9. Merkkola-von Schantz P, Jahkola T, Carpelan A et al (2014) Adverse histopathology and imaging findings in reduction mammaplasty day-surgery patients. Scand J Surg 103:209-214

10. Dupont WD, Page DL (1985) Risk factors for breast cancer in women with proliferative breast disease. $\mathrm{N}$ Engl $\mathrm{J}$ Med 312:146-151

11. Page DL, Dupont WD, Rogers LW et al (1985) Atypical hyperplastic lesions of the female breast. A long-term follow-up study. Cancer 55:2698-2708

12. Blansfield JA, Kukora JS, Goldhahn RT Jr et al (2004) Suspicious findings in reduction mammaplasty specimens: review of 182 consecutive patients. Ann Plast Surg 52:126-130 
13. Carter CL, Corle DK, Micozzi MS et al (1988) A prospective study of the development of breast cancer in 16,692 women with benign breast disease. Am J Epidemiol 128:467-477

14. Dupont WD, Parl FF, Hartmann WH et al (1993) Breast cancer risk associated with proliferative breast disease and atypical hyperplasia. Cancer 71:1258-1265

15. Hartmann LC, Sellers TA, Frost MH et al (2005) Benign breast disease and the risk of breast cancer. N Engl J Med 353:229-237

16. Fitzgibbons PL, Henson DE, Hutter RV (1998) Benign breast changes and the risk for subsequent breast cancer: an update of the 1985 consensus statement. Cancer Committee of the College of American Pathologists. Arch Pathol Lab Med 122:1053-1055

17. Morrow M, Schnitt SJ, Norton L (2015) Current management of lesions associated with an increased risk of breast cancer. Nat Rev Clin Oncol 12:227-238

18. Hage JJ, Karim RB (2006) Risk of breast cancer among reduction mammaplasty patients and the strategies used by plastic surgeons to detect such cancer. Plast Reconstr Surg 117:727-735 discussion 736

19. van der Torre PM, Butzelaar RM (1997) Breast cancer and reduction mammoplasty: the role of routine pre-operative mammography. Eur J Surg Oncol 23:341-342

20. Campbell MJ, Clark CJ, Paige KT (2010) The role of preoperative mammography in women considering reduction mammoplasty: a single institution review of 207 patients. Am J Surg 199:636-640
21. Hennedige AA, Kong TY, Gandhi A (2011) Oncological screening for Bilateral Breast Reduction: a survey of practice variations in UK Breast and Plastics surgeons 2009. J Plast Reconstr Aesthet Surg 64:878-883

22. D'Orsi C, Sickles E, Mendelson E, et al (2013) In: ACR BIRADS Atlas, Breast Imaging Reporting and Data System, Reston, VA

23. Butler CE, Hunt KK, Singletary SE (2003) Management of breast carcinoma identified intraoperatively during reduction mammaplasty. Ann Plast Surg 50:193-197

24. White J, Turton P, Dodwell D et al (2012) Issues in the management of occult neoplasia in breast reduction surgery. Breast $\mathrm{J}$ 18:198-199

25. Houssami N, Abraham LA, Onega T et al (2014) Accuracy of screening mammography in women with a history of lobular carcinoma in situ or atypical hyperplasia of the breast. Breast Cancer Res Treat 145:765-773

26. Saarenmaa I, Salminen T, Geiger U et al (2001) The effect of age and density of the breast on the sensitivity of breast cancer diagnostic by mammography and ultasonography. Breast Cancer Res Treat 67:117-123

27. Narod SA (2012) Breast cancer in young women. Nat Rev Clin Oncol 9:460-470

28. Alvarado R, Lari SA, Roses RE et al (2012) Biology, treatment, and outcome in very young and older women with DCIS. Ann Surg Oncol 19:3777-3784 